\title{
DETERMINACIÓN DEL GRADO DE ACTIVIDAD FÍSICA E INTENCIÓN DE CAMBIO EN NIÑAS Y NIÑOS QUE PARTICIPARON EN LAS ESCUELAS DEPORTIVAS Y RECREATIVAS DEL VERANO DEL 2005 DE LA UNIVERSIDAD DE COSTA RICA
}

\author{
Yamileth Chacón Araya ${ }^{1}$ \\ José Moncada Jiménez ${ }^{2}$
}

\section{Resumen}

El propósito del estudio fue determinar el grado de actividad física y la intención de cambio en niñas, niños y adolescentes matriculadas(os) en un programa de actividad física en la Universidad de Costa Rica.

Las y los participantes (113 sujetos con edades comprendidas entre los 8 y 16 años) respondieron un cuestionario relacionado con la intención de cambio de su actividad física, sus hábitos actuales y las posibilidades de práctica de la actividad física. Los resultados mostraron que el $80.5 \%$ de las niñas y los niños realizaba ejercicio regularmente, al menos durante tres meses consecutivos. Se determinó que el $50.4 \%$ de las y los participantes no tiene la oportunidad de salir a jugar solos, si no los acompañan sus padres o encargados, y el $91.2 \%$ consideraba importante realizar ejercicio junto a sus progenitores. El $94.7 \%$ de las(os) encuestadas(os) recibe clases de Educación Física formales, pero sólo entre una y dos clases semanales,

\footnotetext{
${ }^{1}$ Graduada de la Maestría en Psicopedagogía en la Universidad Estatal a Distancia. Obtuvo la Licenciatura en Educación Física en la Universidad de Costa Rica. Profesora de la Escuela de Educación Física y Deportes, de la Universidad de Costa Rica y coordinadora de Vida Estudiantil de esa unidad académica. Algunos artículos publicados tienen relación con la composición corporal de bailarines costarricenses, el trabajo con cuerdas en niños y la creatividad de jóvenes universitarios. ychacon@cariari.ucr.ac.cr

${ }^{2}$ Cuenta con una Maestría en Ciencias del Movimiento con énfasis en Fisiología del Ejercicio de Springfield College, Massachusetts, USA. Realiza la pasantía del Doctorado en Ciencias de la UCR, en The Ohio State University, Columbus, Ohio, USA. Profesor de la Escuela de Educación Física y Deportes, de la Universidad de Costa Rica. Asesor de tesis de estudiantes de posgrado de la Universidad de Costa Rica y de la Universidad Nacional. Ha publicado numerosos artículos en revistas especializadas y ha sido expositor invitado en congresos nacionales e internacionales. Recientemente se publicó su libro Estadística para ciencias del movimiento humano, editado por la Editorial de la Universidad de Costa Rica.
} 
situación que repercute en su salud física y mental futura y en su posibilidad de incorporar el hábito de ejercitarse en su estilo de vida. Con base en el modelo de las etapas de cambio, se clasificó al $67.3 \%$ de las niñas y los niños, de las(os) cuales un $55.8 \%$ se ubicó en la etapa de mantenimiento.

Este es el primer intento que se realiza en Costa Rica para clasificar a las niñas y los niños utilizando un modelo teórico, y los resultados son congruentes con los reportados en estudios efectuados en otros países. En conclusión, se recomienda el uso de este modelo transteórico para detectar la disponibilidad para el cambio en niñas, niños y adolescentes hacia hábitos y estilos de vida saludables. Es necesario ampliar los parámetros para realizar otras investigaciones al respecto.

\section{Abstract}

The aim of the study was to assess the levels of physical activity and potential for behavior change among children and youth who participated in a structured summer camp program in physical activity and sports at the University of Costa Rica. Participants were 113 subjects with an age range between 8 and 16 years. Subjects responded to a "Stages of Change" questionnaire, and also to items regarding habitual physical activity, and opportunities for becoming physically-active. Statistical analysis showed that $80.5 \%$ of the participants exercised regularly (i.e., for at least for three consecutive months). It was estimated that $50.4 \%$ of the participants did not have the opportunity to play outdoors without parental and/or legal supervision, and that about $91.2 \%$ considered important to exercise along with their parents. From the sample studied, it was found that $94.7 \%$ had consistently received only one or two formal Physical Education classes throughout their lives; which might impact their future physical and psychological health and their possibilities to achieve a desired exercise adherence. Only $67.3 \%$ of the participants were classified in a stage based on the Stages of Change Model for physical activity, with $55.8 \%$ from those belonging to the maintenance stage.

This is the first attempt to investigate the intention to change in Costa $\mathrm{Ri}$ can children; however, the results from the present study are consistent with those reported in other countries. In conclusion, the Transtheoretical Model of Behavior Change might be appropriate to detect disposition to change from detrimental behaviors towards a positive and healthy lifestyle in children and adolescents. Further research is needed to study moderator variables that might impact behavior changes in this population.

\section{Palabras claves}

Modelo transteórico, ejercicio, educación física, actividad física, sedentarismo, niñas y niños. 


\section{Introducción}

Se ha determinado que una niña o un niño sedentaria(o) tiene una alta probabilidad de convertirse en una(un) adulta(o) sedentaria(o) (Fernández et al., 2001). Por esta razón, diversas organizaciones mundiales, como American College of Sports Medicine (2000-2001), realizan esfuerzos para incentivar la actividad física, como un medio para reducir la posibilidad de adquirir enfermedades, entre las que se destacan las cardiovasculares y ciertos tipos de cáncer.

En Costa Rica, los gastos por enfermedades relacionadas con el sedentarismo sobrepasaron, en el año 2003, los $\$ 33.000$ millones, casi el doble de lo que se gastó en el 2002 (Moncada, 2004). En un estudio realizado por la Universidad de Costa Rica y el Ministerio de Educación Pública, desde 1996 y 1998, se demuestra que el 20\% de los estudiantes de la Educación General Básica es sedentario, el sobrepeso oscila entre el 15 y el $23 \%$ y la obesidad entre el 2.2 y el $9.8 \%$ (Fernández y Moncada, 2003).

A pesar de que las campañas para aumentar el grado de actividad física en la población se han enfocado en destacar los beneficios fisiológicos y anatómicos, se estima que se necesita una intervención psicológica para lograr un cambio de comportamiento (United States Department of Health and Human Services, 1996; U.S. Department of Health and Human Services, 2000). Se ha descrito que para pasar de sedentario a físicamente activo, se requiere de un cambio de comportamiento. El estudio del cambio de comportamiento ha sugerido que este es gradual y multifactorial, y que se da por etapas. Así, se han propuesto y estudiado modelos de cambio, entre los cuales se encuentra el modelo transteórico de cambio de comportamiento (Prochaska \& Velicer, 1997).

De acuerdo con Buckworth \& Dishman (2002), el modelo transteórico establece que para que ocurra un verdadero cambio de comportamiento en una persona, esta debe haber pasado por ciertas etapas, entre las que se citan la precontemplación, contemplación, preparación, acción y mantenimiento. El modelo indica que en la etapa de precontemplación, los individuos son inactivos o sedentarios y no tienen intención de cambiar sus hábitos de actividad física en los siguientes 6 meses. En la etapa de contemplación, el individuo es sedentario, pero tiene intención de cambiar en los siguientes 6 meses. En la etapa de preparación, los sujetos son un poco activos, siempre bajo cierto criterio. Por ejemplo, se involucran menos de tres veces por semana en actividades que duran al menos 20 minutos. De acuerdo con este modelo, estas personas tienen la intención de volverse más activas en los próximos 30 días. En la etapa de acción, los sujetos han estado haciendo ejercicio en los últimos 6 meses, pero son los que están en mayor riesgo de dejar de hacerlo. Por último, en la etapa de 
mantenimiento, los sujetos se han estado ejercitando consistentemente por más de 6 meses y la posibilidad de dejar de hacer ejercicio es mucho menor.

Este modelo psicológico establece la disponibilidad para el cambio de un comportamiento; en este caso, pasar de un hábito negativo (ser sedentario) a uno positivo (una vida físicamente activa). A pesar de que el modelo de las etapas de cambio se ha estudiado en poblaciones adultas para cambiar hábitos como el tabaquismo y el alcoholismo (Prochaska \& Velicer, 1997), se cuenta con poca información acerca de su aplicabilidad en el ámbito de la actividad física y el sedentarismo en niñas, niños y adolescentes.

Por ejemplo, en dos estudios de validación del modelo transteórico de cambio de comportamiento realizado entre adolescentes, entre 14 y 18 años, se descubrieron diferencias en la cantidad de ejercicio llevado a cabo por las y los participantes (Lee et al., 2001; Rhodes et al., 2004). Bajo este modelo, se hallaron consistencias en cuanto a la relación de la etapa con la cantidad de ejercicio físico, es decir, a menor etapa (precontemplación, contemplación), menor cantidad de ejercicio físico reportado. De acuerdo con Lee et al. (2001), el modelo presenta una adecuada sensibilidad y especificidad para clasificar a los sujetos, por lo que concluyeron que la validez del instrumento y del modelo como tal son adecuados para estudiar la disposición al cambio en una muestra de adolescentes norteamericanos.

Ante la escasez de estudios en poblaciones jóvenes en el ámbito internacional, el propósito de esta investigación fue determinar el grado de actividad física y la intención de cambio en las niñas, los niños y adolescentes matriculadas(os) en las Escuelas Deportivas y Recreativas del Verano del 2005 (EV-2005), que ofrece la Escuela de Educación Física y Deportes de la Universidad de Costa Rica (UCR).

\section{Metodología}

\section{Sujetos}

Se realizó un estudio transversal, en el que se hizo un muestreo entre las niñas y los niños que se matricularon en las EV-2005 que ofrece cada año la UCR. En total, se matricularon 106 mujeres y 134 hombres $(n=240)$. Aunque la intención inicial de los investigadores era obtener información de toda la población (i.e., censo), solamente se pudo obtener una muestra representativa de 113 participantes, es decir, el $47.08 \%$ del total de niñas y niños matriculadas(os). De la muestra obtenida $(n=113), 50$ eran mujeres $(44.2 \%)$ y 63 hombres $(55.8 \%)$. 
El cuestionario aplicado se sustenta en el modelo transteórico de cambio de comportamiento (Prochaska \& DiClemente, 1983; Buckworth \& Dishman, 2002). La puntuación se obtiene por medio de un algoritmo que se basa en respuestas positivas y negativas. Una persona se encuentra en una etapa de precontemplación si las respuestas a los ítemes del 1 al 4 son negativas. La etapa de contemplación ocurre cuando una persona responde negativamente a los ítemes 1,3 y 4 , y en forma positiva el ítem 2 . Se dice que una persona se encuentra en la etapa de preparación si respondió negativamente a los ítemes 1 y 2 , y de manera positiva en los ítemes 3 y 4 . La etapa de acción ocurre cuando una persona responde positivamente a los ítemes 1,2 y 3 , y en forma negativa al ítem 4. Finalmente, la etapa de mantenimiento se da cuando la persona responde en forma afirmativa a los ítemes del 1 al 4 . La pregunta 5 se usa para determinar si la persona tuvo una recaída, es decir, si dejó el ejercicio por alguna razón. No se incluye en el análisis, sólo se usa como información adicional.

También se incluyeron preguntas para determinar si las niñas y los niños consideraban seguros los parques de juego o "playgrounds", si les gustaba jugar con sus padres, si recibían lecciones de educación física en su institución educativa, y si jugarían más tiempo si sus padres se los permitieran. Adicionalmente, se midió el peso corporal $(\mathrm{kg})$ y la talla $(\mathrm{cm})$ con una balanza electrónica. Con los valores de la talla y el peso corporal se calculó el índice de masa corporal (IMC), una medida que se utiliza en estudios epidemiológicos para clasificar a las personas según su grado de adiposidad. El IMC se calcula dividiendo el peso corporal en kilogramos entre la estatura en metros al cuadrado $\left(\mathrm{kg} / \mathrm{m}^{2}\right)$ (American College of Sports Medicine, 2000, 2001).

\section{Análisis estadístico}

Se obtuvieron promedios y desviaciones estándares como medidas de tendencia central para las variables que así lo ameritaron. Las variables categóricas se describieron por medio de porcentajes. Se utilizaron las pruebas no paramétricas de $X^{2}$ de exactitud de Fisher y la correlación de punto biserial para establecer asociaciones y correlaciones entre las variables categóricas dicotómicas (sexo y la decisión de ejercitarse o no con los padres) y la edad (variable continua). Todos los análisis se llevaron a cabo con el Paquete Estadístico para las Ciencias Sociales $\left(\right.$ SPSS $^{\circledR}$ ), versión 8.0 para Windows ${ }^{\circledR}$. 


\section{Resultados}

En el estudio participaron 113 niñas y niños. Las edades de las y los participantes estuvieron en el rango de los 8 a los 16 años.

La clasificación del grado de adiposidad de las y los participantes se basa en normas costarricenses (Fernández et al., 2001; Fernández Ramírez y Moncada Jiménez, 2003). En la Tabla 1 se presentan los valores de corte para definir sobrepeso y obesidad, así como el porcentaje de sujetos pertenecientes a cada categoría, según su edad y sexo.

El $86.7 \%$ de las y los participantes indicó que actualmente realizaba ejercicio, en contraste con el $11.5 \%$ que dijo lo contrario. Hubo 2 personas (1.8\%) que no respondieron. Para definir ejercicio físico, a las y los participantes se les presentaron ejemplos de ejercicios físicos, entre los cuales se mencionaron actividades como una caminata vigorosa, trotar, nadar, hacer o participar en danza aeróbica, ciclismo, bailes populares y levantar pesas. También se les indicó que no se consideraba como ejercicio físico actividades como boliche, jugar

\section{Gráfico 1.}

Porcentaje de participantes según edad.

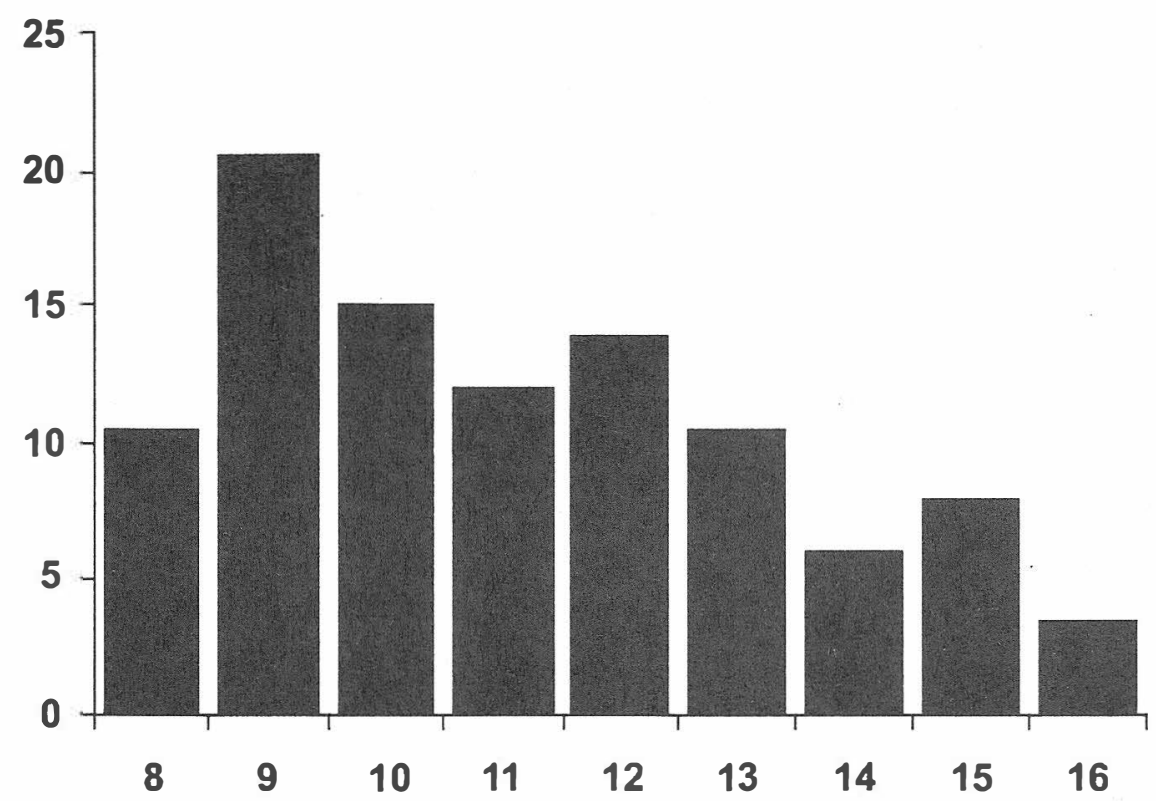

Edad (años) 
Tabla 1.

Grado de adiposidad en las y los participantes de las Escuelas Deportivas y Recreativas de Verano del $\mathbf{2 0 0 5}$ de la Universidad de Costa Rica (EV-2005) en comparación con los valores de referencia para la población costarricense $(n=113)$.

\begin{tabular}{|c|c|c|c|c|c|}
\hline \multirow[b]{2}{*}{ Edad } & \multirow[b]{2}{*}{ Sexo ${ }^{a}$} & \multicolumn{2}{|c|}{ Valores de referencia ${ }^{b}$ delIMC $\left(\mathrm{kg} / \mathrm{m}^{2}\right)$} & \multicolumn{2}{|c|}{ Adiposidad en EV-2005 } \\
\hline & & Sobrepeso $\left(P_{85}\right)$ & Obesidad $\left(P_{95}\right)$ & Sobrepeso(\%) & $\overline{O b e s i d a d}(\%)$ \\
\hline \multirow[t]{2}{*}{8} & $\mathrm{~F}$ & 19.36 & 22.70 & 25 & - \\
\hline & $\mathbf{M}$ & 18.67 & 21.64 & 50 & - \\
\hline \multirow[t]{2}{*}{9} & $\mathrm{~F}$ & 19.86 & 22.87 & 30 & 10 \\
\hline & M & 19.07 & 21.80 & 23 & 10 \\
\hline \multirow[t]{2}{*}{10} & $\mathrm{~F}$ & 21.16 & 24.64 & 25 & - \\
\hline & M & 21.06 & 25.54 & 11 & 22 \\
\hline \multirow[t]{2}{*}{11} & $\mathrm{~F}$ & 22.26 & 25.34 & 28.5 & - \\
\hline & M & 21.08 & 24.36 & - & 50 \\
\hline \multirow[t]{2}{*}{12} & $\mathrm{~F}$ & 23.42 & 26.61 & - & - \\
\hline & M & 21.61 & 25.24 & 33 & - \\
\hline \multirow[t]{2}{*}{13} & $\mathrm{~F}$ & 23.52 & 26.24 & - & - \\
\hline & $\mathrm{M}$ & 22.41 & 25.76 & 25 & - \\
\hline \multirow[t]{2}{*}{14} & $\mathrm{~F}$ & 24.34 & 27.22 & & \\
\hline & $\mathrm{M}$ & 22.98 & 26.66 & 16.6 & 16.6 \\
\hline \multirow[t]{2}{*}{15} & $\mathrm{~F}$ & 24.60 & 28.00 & 16.6 & - \\
\hline & $\mathbf{M}$ & 23.00 & 26.04 & 25 & - \\
\hline \multirow[t]{2}{*}{16} & $\mathrm{~F}$ & 25.22 & 27.43 & 25 & - \\
\hline & M & 23.39 & 25.75 & - & - \\
\hline
\end{tabular}

Notas:

${ }^{\text {a }}$ Sexo: $\mathrm{F}=$ femenino; $\mathrm{M}=$ masculino.

${ }^{b}$ Fuente: Fernández Ramírez, A. y Moncada Jiménez, J. (2003). "Obesidad y sobrepeso en la población estudiantil costarricense entre los 8 y 17 años". Revisea Costarricense de Ciencias Médicas, 24(3,4), 95-113.

cartas (naipe) ni ajedrez. El 84.1\% de los sujetos respondió que pretendía hacer ejercicio físico en los siguientes 6 meses, mientras que el $13.3 \%$ de las y los participantes respondió que no pretendía realizar ejercicio físico. Tres sujetos (2.7\%) no respondieron.

Se encontró que el $80.5 \%$ de las y los participantes realizaba ejercicio regularmente. Se definió "regularmente" hacer una actividad física al menos 3 
veces por semana, con una duración de por lo menos 20 minutos cada sesión. De esta forma, el $17.7 \%$ de las y los participantes respondió que no hacía ejercicio en forma regular.

El $68.1 \%$ de las y los participantes indicó que había realizado ejercicios físicos con regularidad anteriormente, en contraste con el $31 \%$ que manifestó lo contrario. Hubo un participante $(0.9 \%)$ que no respondió. Se encontró que el $76.1 \%$ de las encuestadas y los encuestados había hecho ejercicio físico otras veces, al menos durante 3 meses seguidos. Sin embargo, el 23\% indicó lo contrario y una persona $(0.9 \%)$ no respondió.

A las y los participantes se les preguntó si harían más ejercicio si sus padres los dejaran ir a jugar solos. Las opiniones fueron muy similares, es decir, un $50.4 \%$ respondió favorablemente, mientras que el $47.8 \%$ dijo lo contrario. Hubo 2 personas $(1.8 \%)$ que no respondieron la pregunta.

La mayoría de las encuestadas y los encuestados (71.7\%) dijo que no consideraba que los parques de juego o "playgrounds" fuesen peligrosos. Sin embargo, el $26.5 \%$ sí pensaba que lo eran, mientras que 2 personas $(1.8 \%)$ no respondieron.

El $94.7 \%$ de las y los participantes recibe clases de educación física, 4 sujetos no $(3.5 \%)$ y 2 sujetos $(1.8 \%)$ no respondieron. De las y los participantes que manifestaron recibir clases de educación física, se pudo determinar que más del $80 \%$ recibe sólo entre una y dos clases semanales y 3 sujetos $(2.7 \%)$ manifestaron recibir educación física 4 días por semana. Suponiendo que una clase efectiva de educación física puede durar 20 minutos, se podría estimar que el grado de actividad física semanal atribuible sólo a la clase obligatoria de educación física es, para este grupo de niñas y niños y en el mejor de los casos, de 80 minutos (4 días x 20 minutos/clase).

Se encontró que al $8.8 \%$ de las niñas y los niños $(n=10)$, no le gusta hacer ejercicio con sus padres; en contraste con el $91.2 \%$ que respondió que sí le gusta ejercitarse en compañía de sus padres. La prueba de exactitud de Fisher indicó que no existe una asociación entre el sexo de las y los participantes y la opinión a favor o no de ejercitarse con sus padres $\left(X^{2}=0.91\right.$, g.l. $\left.=1, p>.05\right)$. Por medio de una correlación de punto biserial, tampoco se encontró una asociación significativa entre la edad de las y los participantes y la decisión de ejercitarse o no con los padres $\left(r_{b}=-0.08, p>0.05\right)$.

El modelo de las etapas de cambio permitió clasificar al $67.3 \%$ de los sujetos en las etapas de precontemplación (0.9\%), contemplación $(0.9 \%)$, acción (9.7\%) y mantenimiento (55.8\%). No se logró clasificar al $32.7 \%$ de las encuestadas y los encuestados, debido a que el algoritmo recomendado no lo permitía o porque no respondieron esa sección del cuestionario. 


\section{Discusión}

El propósito de este estudio fue determinar el grado de actividad física y la disposición para el cambio de comportamiento en niñas, niños y adolescentes que asistieron a las EV-2005 de la UCR, que forman parte de un programa de extensión hacia la comunidad. Este es el primer estudio, del cual los investigadores tienen conocimiento, en el que se evalúan las intenciones de cambio hacia la actividad física en niñas y niños costarricenses.

En las EV-2005 de la UCR se atiende, en su mayoría (i.e., > 70.8\%), a niñas y niños entre 8 y 12 años de edad. Esta tendencia en la matrícula concuerda con un menor grado de participación en actividades físicas, observado especialmente en las mujeres, a medida que se acercan a la adolescencia (Fernández Ramírez y Moncada Jiménez, 2003). El grado de reducción en la actividad física se relaciona con un aumento en el grado de sobrepeso y obesidad. Con base en las normas costarricenses para definir obesidad y sobrepeso (Fernández Ramírez y Moncada Jiménez, 2003), se encontraron niveles de sobrepeso y obesidad en el rango del 10 al 50\%. Queda en evidencia la necesidad de continuar promoviendo los programas deportivos y recreativos a través de las escuelas de verano, de manera que las niñas y los niños puedan tener acceso a actividades físicas durante el periodo de vacaciones escolares.

Aunque más del $85 \%$ de las y los participantes indicó que realizaba ejercicio en ese momento, se encontró, consistentemente, que al menos el $10 \%$ de las encuestadas y los encuestados no hacía ejercicio alguno, que no pretendía realizar ejercicio en el siguiente semestre y que nunca había hecho ejercicio regularmente. Este grupo de personas puede clasificarse como sedentario crónico y presenta el mayor potencial de convertirse en adultos obesos (Fernández et al., 2001).

Las y los participantes se mostraron optimistas respecto a sus pretensiones de realizar ejercicio físico en los siguientes 6 meses. Cabe recordar que las escuelas deportivas de verano que ofrece la UCR coinciden con el inicio del año; es decir, estas comienzan en la segunda semana de enero y finalizan en la segunda semana de febrero. Las niñas y los niños han tenido "vacaciones escolares" durante al menos 30 días y es posible que sus expectativas también incluyeran las clases de educación física que eventualmente recibirían al inicio del año escolar; no obstante, esto no se pudo cuantificar con el instrumento utilizado para este estudio.

En este estudio se les preguntó a las niñas y los niños si harían más ejercicio si sus padres las(os) dejaran jugar solas(os), es decir, sin supervisión. La interpretación de las respuestas a esta pregunta es complicada. Por una parte, se 
puede pensar que las niñas y los niños no tienen más opción que ir a las escuelas de verano, porque sus padres utilizan este servicio como una especie de "guardería". Por otra parte, puede ser que las niñas y los niños en realidad sean físicamente activas(os), pero ya no quieren realizar más actividades físicas. Algunas(os) estudiantes, posiblemente, participan en actividades extraescolares, como por ejemplo, clases particulares de deportes (natación, tenis o gimnasia), razón por la que se pueden sentir tensas(os), fatigadas(os), deprimidas(os) e incluso sobreentrenadas(os); por este motivo, no pretenden realizar más actividades físicas (Committee on Sports Medicine and Fitness and Committee on School Health, 2001).

Es indudable que queda aún por determinar cuáles son los motivos para desear o evitar realizar ejercicio físico en esta población particular.

Con respecto a los parques de juego o "playgrounds", existe una serie de estudios en los que se demuestra que el espacio físico para la recreación en el vecindario o barrio puede influir en la salud emocional y física de las(os) residentes (Sallis et al., 2001). En este estudio, la mayoría de las niñas y los niños expresó que los parques de juego eran sitios seguros; o mejor dicho, no eran sitios peligrosos. Ante la inseguridad ciudadana que se vive en Costa Rica, todavía queda por realizar más investigación acerca de la seguridad en los parques de juego y de las posibles acciones para que las personas utilicen más estos espacios de recreación, de manera que puedan acceder a sitios seguros, limpios y con materiales en buen estado, que sirvan para el esparcimiento de diferentes grupos etáreos y en los que se reduzcan los riesgos de sufrir accidentes ${ }^{3}$.

En este estudio, se estimó el grado de actividad física atribuible a las clases de educación física y se encontró que, en el mejor de los casos, una niña o un niño realiza aproximadamente 80 minutos de actividades físicas semanales. Esta cantidad es inaceptable, ya que los organismos internacionales recomiendan realizar, al menos, 30 minutos diarios de actividad física, para un estimado semanal mínimo de, al menos, 120 minutos (American College of Sports Medicine, 2000, 2001).

Aunque existen diversos factores que influyen en el grado de actividad física en las niñas y los niños, en este estudio se quiso determinar si los padres podrían estimular la práctica de actividades conjuntas. Los motivos de las niñas y los niños para ejercitarse o no con sus padres requieren de mayor investigación. Por el momento, el estudio halló que no existe una asociación entre la edad o el sexo de las encuestadas y los encuestados, con la preferencia de ejercitarse con sus padres.

\footnotetext{
${ }_{3}^{3}$ Por ejemplo, los trabajos de Bond \& Peck, 1993; Centers for Disease Control and Prevention, 1999; Cradock et al., 2005; Macarthur et al., 2000; Mack, Hundson \& Thompson, 1997; Mott et al., 1997; Salazar Salas, 2000.
} 
Sería importante proponer algún curso piloto recreativo o deportivo en el que se involucren tanto madres y padres como niñas y niños y estudiar las interacciones entre estas y estos y sus progenitores. Se han elaborado programas para niñas y niños de edad temprana donde los padres tienen un papel fundamental para estimular el movimiento de sus hijas o hijos, pero sobre todo, para propiciar una relación más cercana, que mejore el canal socioafectivo. A estos programas se les ha llamado estimulación temprana y matrogimnasia. Para Muñoz (1996), la estimulación temprana es un proceso natural de desarrollo, manejado en forma de juego, que se puede poner en práctica al relacionarse diariamente con la o el bebé de 0 a los 12 meses. Se indica también como uno de los objetivos de la matrogimnasia el de propiciar, mediante las actividades físicas, la madurez emocional de la niña y el niño con influencia de su madre, con el fin de darle mayor confianza y seguridad. Esta actividad se ubica, principalmente, en el periodo preescolar (Herrera et al., 1987).

Para un buen porcentaje de niñas y niños del presente estudio, es importante la compañía de los padres para ejercitarse o jugar. Por lo general, los padres de familia o encargadas(os) cumplen un papel más pasivo en este programa de verano, porque sólo dejan a las niñas y los niños y luego regresan por ellas y ellos al final del periodo de clases, o en algunos casos, los esperan de una a tres horas en las instalaciones, sin la disposición de ejercitarse durante ese tiempo. Se considera que para llevar a cabo un programa piloto se deben tomar en cuenta las condiciones (tiempo, interés) de las madres y los padres para incorporarse a este programa y la posibilidad de integrar uno o varios cursos con estas características, con el fin de evaluar los resultados.

Sin embargo, habría que pensar en la posible aceptación de un tipo de programa de esta naturaleza en preadolescentes y adolescentes, quienes se encuentran en una etapa de transición hacia la independencia de sus padres. A pesar de ello, al tomar en cuenta niñas y niños de edades mayores, puede que, mediante un programa integrador, se favorezca la relación generacional y se logre un mayor apoyo para ambas partes, encontrándose más dispuestas(os) en su esfuerzo por habituarse a la práctica física y deportiva.

El modelo transteórico de cambio de comportamiento se utilizó para establecer la disposición de los individuos por cambiar hábitos negativos o estimular hábitos positivos. Este modelo se ha utilizado en otras poblaciones para estudiar el comportamiento hacia el cambio, por ejemplo, en fumadoras(es) y drogadictas(os) o para estimular el uso de servicios médicos, como mamografías, la reducción de peso, el uso del preservativo y el cambio hacia dietas saludables (Prochaska \& Velicer, 1997; Spencer, 2002). 
Como ya se mencionó, el modelo ha sido poco estudiado en poblaciones de niñas, niños y adolescentes ${ }^{4}$. No obstante, de manera similar al presente estudio, se han reportado porcentajes bajos en las etapas de precontemplación y contemplación, porcentajes moderados en las etapas de preparación y acción y porcentajes altos en la etapa de mantenimiento. Sin embargo, en el presente estudio no se logró clasificar a ningún participante en la etapa de preparación y tampoco fue posible clasificar al $32.7 \%$ de las y los participantes. El rango de edad de los sujetos que participaron en el estudio de Nigg \& Courneya (1998) fue de 13 a 19 años $(n=819)$, similar al estudio de Prapavessis et al. (2004), quienes utilizaron una muestra de jóvenes entre los 14 y 19 años $(n=3972)$.

Por su parte, Cardinal et al. (1998) estudiaron específicamente a un grupo de preadolescentes $(n=669)$, cuya edad promedio era de 8 años (rango de 5 a 11 años). La muestra fue casi exclusivamente de escolares blancos (94.2\%) de escuelas suburbanas del medio oeste estadounidense. Los investigadores clasificaron a la mayoría de los sujetos $(87.3 \%)$ en las etapas de mayor actividad física (acción y mantenimiento). En el presente estudio, solamente se pudo clasificar, en esas dos etapas, al $65.5 \%$ de las encuestadas y los encuestados. A pesar de esta diferencia, en esta investigación se utilizó una definición escrita de

\section{Gráfico 2.}

Clasificación de niñas, niños y adolescentes de Costa Rica, en las etapas de cambio según el modelo transteórico de cambio de comportamiento.

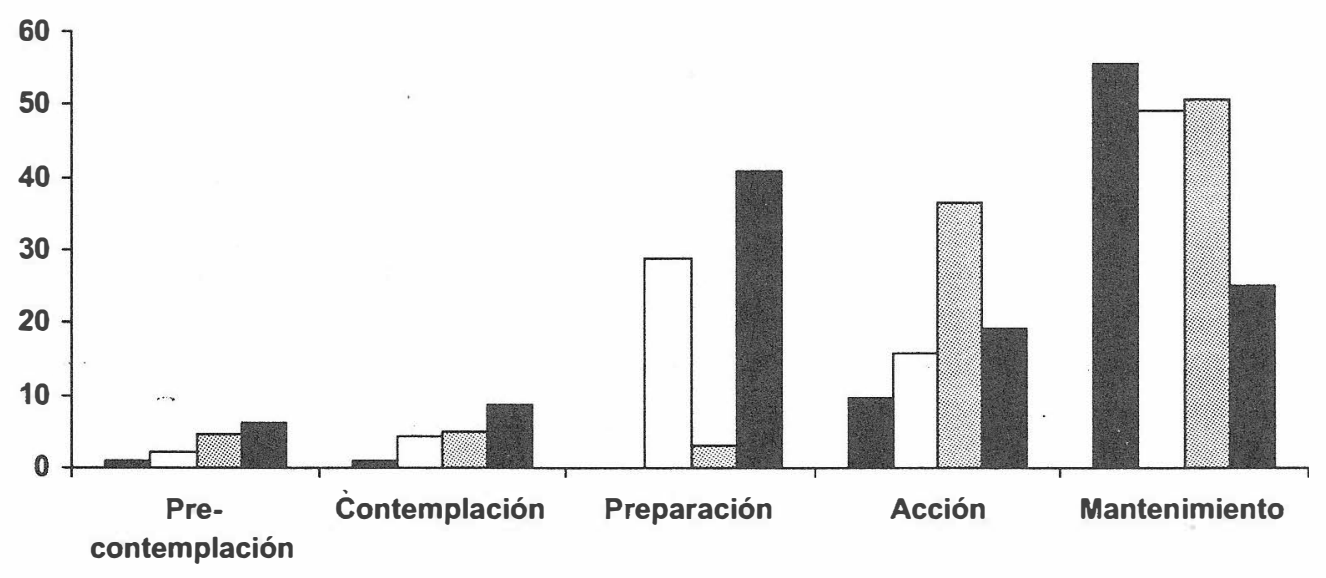

Etapas de Cambio

\footnotetext{
${ }^{4}$ Algunos de estos autores son: Cardinal et al., 1998; Hausenblas et al., 2002; Nigg \& Coumeya, 1998; Maddison et al., 2002; Prapavessis et al., 2004.
} 
los términos "actividad física" y "regularidad" del ejercicio; aspecto que no consideraron Cardinal et al. (1998) y que reconocen en la discusión de sus resultados como un factor que puede mejorar la clasificación de las y los participantes a las etapas de cambio.

Otras(os) investigadoras(es) han intentado cuantificar qué tan potente es este modelo para explicar cambios de comportamiento en niñas, niños y adolescentes. De acuerdo con Nigg (2003), el modelo transteórico apenas explica una parte de la varianza total de un comportamiento, en este caso, el ejercicio físico o la adherencia al ejercicio. Para estudiar si la motivación de practicar un deporte podía explicar un porcentaje de la varianza, Nigg diseñó un estudio longitudinal de tres años en el que participaron 819 adolescentes entre los 13 y 15 años de edad. De los 819 participantes iniciales, sólo pudo obtener datos completos de 400 sujetos (48.84\%) y encontró que la participación en deportes no era un factor importante que pudiera explicar la varianza más allá de lo que el modelo transteórico era capaz de mostrar.

En conclusión, con este estudio se logró determinar el grado de actividad física de las niñas y los niños que participaron en las EV-2005 de la UCR, y su intención de cambio de conducta según el modelo transteórico. Con base en los resultados expuestos, se concluye que el modelo transteórico se podría seguir utilizando para detectar la disponibilidad para el cambio en niñas, niños y adolescentes hacia hábitos y estilos de vida saludables (ejercicio físico, apropiada alimentación). Sin embargo, se recomienda revisar el cuestionario, puesto que una tercera parte de las y los participantes no pudo clasificarse en alguna de las etapas que propone el modelo. Como tal, este modelo se debe someter a prueba, por medio de más estudios con muestras representativas de la población escolar. Además, se deben tomar en cuenta variables moderadoras de la adopción de un hábito en dicha población, como por ejemplo, el estrato socioeconómico, el tamaño familiar, el grado de escolaridad de las madres y los padres, la cercanía y nivel de seguridad de los parques de juego, entre otras. 


\section{Referencias}

American College of Sports Medicine. (2000). ACSM's guidelines for exercise testing and prescription $\left(6^{\text {th }}\right.$ Ed.). Baltimore, MD: Lippincott, Williams \& Wilkins.

American College of Sports Medicine. (2001). ACSM's resource manual for guidelines for exercise testing and prescription ( $4^{\text {th }}$ Ed.). Baltimore, MD: Lippincott, Williams \& Wilkins.

Aveyard, P., Sherratt, E., Almond, J., Lawrence, T., Lancashire, R., Griffin, C., \& Cheng,

K. K. (2001). The change-in-stage and updated smoking status results form a cluster-randomized trial of smoking prevention and cessation using the transtheoretical model among British adolescents. Preventive Medicine, 333, 313-324.

Bond, M. T. \& Peck, M. G. (1993). The risk of childhood injury on Boston's playground equipment and surfaces. American Journal of Public Health, 83, 731-733.

Buckworth, J. \& Dishman, R. K. (2002). Exercise psychology. Champaign, IL: Human Kinetics.

Cardinal, B. J., Engels, H. J., \& Zhu, W. (1998). Application of the Transtheoretical Model of Behavior Change to preadolescent's physical activity and exercise behavior. Pediatric Exercise Science, 10, 69-80.

Centers for Disease Control and Prevention. (1999). Playground safety-United States, 1998-1999. Morbidity and Mortality Weekly Report, 48(16), 329-332.

Committee on Sports Medicine and Fitness and Committee on School Health. (2001). Organized sports for children and preadolescents. Pediatrics, 107, 1459-1462. Cradock, A. L., Kawachi, I., Colditz, G. A., Hannon, C., Melly, S. J., Wiecha, J. L., \& Gortmaker, S. L. (2005). Playground safety and access in Boston neighborhoods. American Journal of Preventive Medicine, 28(4), 357-363.

Ellaway, A. \& Macintyre, S. (1996). Does where you live predict health related behaviors? A case study in Glasgow. Health Bulletin (Edinburgh), 54, 443-446.

Fernández, A., González, C., Moncada, J., Pearson, G., Picado, M. y Salas, R. (2001). Normas nacionales. Componentes de salud física: estudiantes costarricenses entre 8 y 17 años. San José, Costa Rica: Editorial Baula.

Fernández Ramírez, A. y Moncada Jiménez, J. (2003). Obesidad y sobrepeso en la población estudiantil costarricense entre los 8 y 17 años. En: Revista Costarricense de Ciencias Médicas, 24(3,4), 95-113.

Hausenblas, H. A., Nigg, C. R., Downs, D. S., \& Connaughton, D. P. (2002). Perceptions of exercise stages, barrier self-efficacy, and decisional balance for middleschool students. Journal of Early Adolescence, 22(4), 436-454.

Herrera, L., Cortés, M., Herrera, R. y Arreguín, N. (1987). Matrogimnasia. México, D.F.: Editorial Didáctica Moderna, S.A.

Lee, R. E., Nigg, C. R., DiClemente, C. C., \& Courneya, K. A. (2001). Validating motivational readiness for exercise behavior with adolescents. Research Quarterly for Exercise and Sport, 72(4), 401-410. 
Lidstone, J. E., Amundson, M. L., \& Amundson, L. H. (1991). Depression and chronic fatigue in the high school student and athlete. Primary Care, 18(2), 283-296.

Macarthur, C., Hu, X., Wesson, D., \& Parkin, P. (2000). Risk factors for severe injuries associated with fall from playground equipment. Accident Analysis \& Prevention, 32(3), 377-382.

Mack, M. G., Hundson, S., \& Thompson, D. (1997). A descriptive analysis of children's playground injuries in the United States 1990-4. Injury Prevention, 3, 100-103.

Maddison, R., Brading, F., \& Prapavessis, H. (2002). Predicting exercise stage transition in an adolescent New Zealand population: a test of the transtheoretical model. Joumal of Science and Medicine in Sport (Canberra, Aust.), 5(4 Suppl.), 94.

Moncada, J. (4 de abril del 2004). Un gran cambio nacional. En: Periódico La Nación, Sección Opinión, p. 30-A.

Mott, A., Rolfe, K., James, R., Evans, R., Kemp, A., Dunstan, F., Kemp, K., \& Sibert, J. (1997). Safety of surfaces and equipment for children in playgrounds. Lancet, 349(9069). 1874-1876.

Muñoz, C. M. (1996). Lo mejor para estimulación temprana. Colombia: AUnoA Editores.

Nigg, C. R. (2003). Do sport participation motivations add to the ability of the Transtheoretical Model to explain adolescent exercise behavior? International Journal of Sport Psychology (Rome), 34(3), 208-225.

Prapavessis, H., Maddison, R., \& Brading, F. (2004). Understanding exercise behavior among New Zealand adolescents: A test of the Transtheoretical Model. Joumal of Adolescent Health, 35(2), 346e.17-346.e27.

Prochaska, J. O. \& Velicer, W. F. (1997). The Transtheoretical Model of Health Behavior Change. American Joumal of Health Promotion, 12(1), 38-48.

Rhodes, R. E., Berry, T., Naylor, P. J., \& Higgins, S. J. W. (2004). Three-step validation of exercise processes of change in an adolescent sample. Measurement and Evaluation in Physical Education and Exercise Science, 8(1), 1-20.

Salazar Salas, C. G. (2000). ¿Negligencia en la seguridad de los parques de juego? En: Revista Costarricense de Salud Pública, 9(17), 1-9.

Sallis, J. F., Conway, T. L., Prochaska, J. J., McKenzie, T. L., Marshall, S. J., \& Brown, M. (2001). The association of school environments with youth physical activity. American Journal of Public Health, 91(4), 618-620.

Scanlan, T. K. \& Lewthwaite, R. (1988). From stress to enjoyment: Parental and coach influences on young participants. Brown, E. W. \& Branta, C. F. (Eds.), Competitive sports for children and youth. An overview of research and issues. Champaign, IL: Human Kinetics.

Spencer, L. (2002). The change-in-stage and updated smoking status results from a cluster-randomized trial of smoking prevention and cessation using the transtheoretical model among British adolescents. American Joumal of Health Promotion, 17(1), 82-83. 
U.S. Department of Health and Human Services. (2000). Healthy People 2010: Understanding and Improving Health ( $2^{\text {nd }}$ Ed.). Washington, D.C.: U.S. Government Printing Office.

United States Department of Health and Human Services. (1996). Physical activity and health. A report of the Surgeon General. Atlanta, GA: U.S. Department of Health and Human Services, Center for Disease Control and Prevention, National Center for Chronic Disease Prevention and Health Promotion, The President's 


\section{Anexo 1}

\section{Cuestionario de las Etapas de Cambio - Niños y Adolescentes}

Este cuestionario es anónimo, por lo tanto no debe escribir su nombre. Por favor, lea cuidadosamente las preguntas que aparecen a continuación, y responda de la manera más sincera posible. -¡Gracias!

Edad: años

Es usted: una mujer

un hombre

Estatura en metros:

Peso kilogramos:

Ahora, responda las siguientes preguntas acerca del ejercicio físico:

Ejercicio físico incluye actividades como una caminata vigorosa, trotar, nadar, danza aeróbica, ciclismo, bailes populares, levantar pesas, etc. En este cuestionario no se considera como ejercicio físico actividades como boliche, jugar cartas (naipe), ni ajedrez.

Por favor marque con una " $\mathrm{X}$ " la casilla que mejor describa su opinión:

1. Actualmente yo hago ejercicio

2. Pretendo empezar a hacer ejercicio en los próximos 6 meses

3. Actualmente yo hago ejercicio regularmente

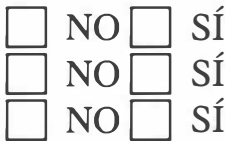

"regularmente" significa hacer la actividad 3 veces o más por semana, con una duración de por lo menos 20 minutos cada sesión.

4. Yo he hecho ejercicio regularmente en los últimos 6 meses

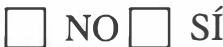

5. En otros momentos, yo he hecho ejercicio regularmente al menos 3 meses seguidos

6. Yo haría más ejercicio si mis papás me dejaran ir a jugar solo

7. Los parques o playas son peligrosos para hacer ejercicio

8. Yo recibo clases de educación física

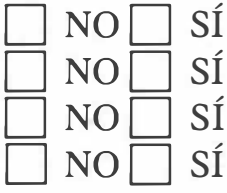

¿Cuántas veces a la semana recibe clases de educación física?

9. Me gusta ir a hacer ejercicio o a jugar con mis papás

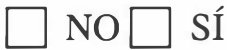

Aquí se termina la encuesta. Muchas gracias por su cooperación. 